\title{
RASGOS DE COLONIALIDAD EN LA ATENCIÓN MÉDICA A PUEBLOS INDÍGENAS
}

COLONIALITY FEATURES IN

MEDICAL CARE FOR INDIGENOUS PEOPLES

Mariana Ramírez Manzano

ORCID 0000-0001-7908-198X

Universidad Autónoma de la Ciudad de México

Ciudad de México, México

\section{Resumen}

El centro de interés de este artículo es la atención médica hospitalaria a los pueblos indígenas en la Colonia y los fundamentos, prácticas, relaciones interétnicas (entendiendo por etnia igualmente un pueblo mesoamericano y cualquier pueblo ajeno a Mesoamérica). Se aludirá a algunas características de la medicina y práctica médica indígena a fin de destacar las similitudes y diferencias y las actitudes de las etnias europeas hacia tales contrastes. El propósito de ejemplificar las prácticas médicas de una y otra etnia y contrastar sus respuestas es encontrar en la analogía una razón suficiente para cuestionar el posicionamiento de una práctica sobre la otra. Plantear los rasgos favorables o desfavorables para la atención médica a los pueblos indígenas en la Colonia para finalmente ubicar la presencia actual de dichos rasgos en algún caso.

Palabras clave: Hospitalización en la Conquista, Medicina indígena, Nueva España.

\section{Resumo}

O centro de interesse desse artigo é a assistência médica hospitalar aos povos indígenas da colônia e os fundamentos, práticas, relações interétnicas (etnia também significa um povo mesoamericano e qualquer pessoa fora da Mesoamérica). Algumas características da medicina indígena e da prática médica também serão mencionadas, a fim de destacar as semelhanças e diferenças e atitudes dos grupos étnicos europeus em relaçáo a esses contrastes. $\mathrm{O}$ objetivo de exemplificar as práticas médicas de ambos os grupos étnicos e contrastar suas respostas é encontrar na analogia uma razão suficiente para questionar o posicionamento

\section{Abstract}

The focus of this paper is hospital medical care for indigenous peoples in the colony during the Spanish conquest and their main bases, practices and inter-ethnic relations (we understand ethnicity as also including Mesoamerican people and any other people outside Mesoamerica). Some characteristics of indigenous medicine and medical practice will also be discussed in order to highlight the similarities, differences, and attitudes of European ethnic groups towards such contrasts. The purpose of exemplifying the medical practices of both ethnic groups, and contrasting their responses, is to find in the analogy a sufficient reason to question 
de uma prática sobre a outra. Levantar as características do colonialismo favorável na assistência médica aos povos indígenas da Colônia e o desfavorável para garantir a saúde.

Palavras-chave: Hospitalização na Conquista, Medicina Indígena, Nova Espanha. the positioning of one practice over the other, as well as to highlight positive and negative aspects of the medical care to indigenous peoples during the colonial period in order to identify if any of these features are still prevalent in any cases.

Keywords: Hospitalization in the Conquest, Indigenous Medicine, New Spain.

Este artículo tiene como guía la propuesta planteada por Verdesio en su inflexión subalternista de los estudios coloniales, la cual subraya la pertinencia de observar las formas de imposición del capitalismo y de identificar los procesos gestados en la colonia que se reproducen y se actualizan en "situaciones de opresión y desigualdad" (VERDESIO, 2018, p. 100).

En este sentido, hacemos un acercamiento a los procesos de atención médica y atención hospitalaria a los pueblos indígenas en la Nueva España. El lector encontrará una selección de fuentes relativas a las prácticas médicas en Europa y su traslado a los territorios colonizados con similitudes y particularidades ligadas a la noción de superioridad que los colonizadores asumieron frente a las culturas desconocidas por ellos.

Se presentan algunas referencias al establecimiento de hospitales y los actores involucrados en su constitución, así como los diversos intereses y perspectivas que pudieron motivar su fundación, características y funcionamiento. Aludimos a órdenes religiosas, médicos europeos y médicos mesoamericanos. También, expondremos el tipo de prácticas médicas que tenían lugar en los pueblos colonizados, con propensión a trazar los rasgos comunes y la capacidad o incapacidad de los implicados para reconocer y aprovechar lo diferente en favor de la salud. Destacaremos cómo, durante la Colonia, algunos estudiosos hicieron esfuerzos por comprender y reconocer las aportaciones médicas de los pueblos, cómo otros utilizaron su interpretación de inferioridad para utilizar los cuerpos como objetos de conocimiento para la medicina y mostraremos ejemplos de la interacción mutua entre saberes, muy pocos y muy aislados.

Lo que de verdad prevalece y hasta podríamos decir que se mantiene es esa mirada lateral, de reojo, de soslayo de la medicina europea sobre la no-europea, que es la que se mantiene actualmente en los programas de formación médica. 
En cambio, si se ofreciera la información sin el dejo colonial, y se presentaran en condición de igualdad los conocimientos médicos de las diferentes culturas, se promovería la identificación de conocimientos que llevarían al objetivo compartido entre las medicinas: la efectividad del remedio.

\section{Medicina en Europa y medicina en Mesoamérica}

Es cardinal señalar que el punto de partida de la medicina y la atención médica en la Nueva España fue la ciencia desarrollada en Inglaterra, Italia, Suiza, sitios donde esta ciencia cobró su mayor auge y aportaciones relevantes para la comprensión del cuerpo, la enfermedad y la salud. Se van abordando las concomitancias entre las prácticas médicas europeas y las mesoamericanas. ${ }^{1}$

En el mundo médico europeo de siglo XVII, la naturaleza de una enfermedad se determinaba con las declaraciones y la descripción del paciente ante el galeno, con la observación de la apariencia y el comportamiento del enfermo, mas no se acostumbraba una auscultación ni revisión del cuerpo, ya que se consideraba indigno de una profesión intelectual el realizar un trabajo manual. El tema de las afirmaciones del paciente fue considerado por algunos médicos un impedimento para un correcto diagnóstico, ya que conocieron casos en los que el enfermo había omitido indicar algún acontecimiento o antecedente determinante de su enfermedad, y el médico, al no contar o indagar por mayor información, no podía incluir esa variable en el diagnóstico y, por lo tanto, en el tratamiento. (REISER, 1990, p. 19)

Especialmente, la queja de un médico hace alusión a la dificultad para obtener, de las personas pobres, un testimonio completo de lo que implica su malestar. Llama la atención que se refiera a las personas pobres y no a todas. Seguramente, los padecimientos se habían desarrollado mucho antes de que el paciente llegara a la atención médica y, al acudir con el doctor, ya no recuerda los acontecimientos que influyeron en él. Si a la dificultad para obtener un testimonio completo agregamos la dificultad para comprender un idioma distinto, ya podemos imaginar el entuerto de dar atención médica a los pueblos indígenas en la Colonia y, desde luego, hasta la actualidad.

Por lo que toca a la exploración física del paciente, como se mencionó anteriormente, se tenía por cosa indigna para los médicos, ya que se suponía que el trabajo manual implicado en la auscultación denigraba el prestigio del médico formado en textos reconocidos y venerados. Lo anterior se remonta

1 A decir de Pedro A. Serrano: "Es médico (...) no sólo el que repara un daño o alivia un mal; sino sobre todo aquel que preserva la integridad humana y, para ello, intenta comprenderla. Este intento, este pretender abarca todo lo humano, es lo que hace indispensable para el médico empaparse de la cultura, la profesión se enaltece y permite alcanzar un sano equilibrio frente al creciente pesos de la tecnificación". (GARCÍA, 1994, p. 166) A partir del sentido en que este médico moderno comprende su profesión, buscamos algunos ejemplos de cómo se logró o se impidió este conocimiento de la cultura. 
al siglo XIII cuando las universidades no daban importancia al aprendizaje basado en la observación de la naturaleza, práctica recurrente en las facultades de medicina y en el resto de la universidad. Por el contrario, se subrayaba el enfoque teórico y filosófico del aprendizaje. De este modo, la educación médica, como lo dice el historiador moderno Charles Talbot (1970), se había convertido en "un proceso de aprender un cuerpo fijo de doctrina, y luego amplificarla y manipularla por medio del análisis sutil y el argumento dialéctico". Los médicos que se dedicaban a las actividades manuales eran criticados "por la suposición de que la dignidad de la medicina sólo quedaría asegurada mediante una preocupación por las ideas universales". La amenaza del trabajo manual a la categoría y, a la postre, al bolsillo, hacía que los médicos se retiraran de él "como de una peste", notó categóricamente Vesalius, "no fuera a ser que los rabinos (las autoridades principales) de la medicina los desprestigiaran ante la masa ignorante, como barberos, y adquieran menos riqueza y honores". (REISER, 1990, p. 24)

La afirmación del médico de Padua, Andreas Vesalius obedece a un trabajo sistemático de autopsias mediante el cual adquirió un conocimiento para la clínica, acerca de la salud y la enfermedad. Su método de conocimiento rompió con la condena al trabajo manual en la medicina y aportó a la comprensión de los procesos de salud y enfermedad reflejados en el cuerpo mediante la observación directa. De ella, resultó la obra De humani corporis fabrica (1543) (REISER, 1990, p. 23)

Comparó a los lectores con unos "grajos en lo alto de sus cátedras, con una egregia arrogancia, graznando cosas que nunca han investigado sino simplemente confiado en la memoria, a partir de los libros de otros, o leyendo lo que ya está descrito".

La Fabrica (...) anunció un nuevo principio de descubrir los hechos y someterlos a prueba de verdad en cuestiones de anatomía: todos los planteamientos y las hipótesis anatómicas debían quedar sometidas a una metódica revisión, por medio de la disección y observación de cadáveres humanos, proceso caracterizado por el historiador C.D. O'Malley, como una "congruente política de la duda". (REISER, 1990, p. 26)

Por esta ruta, se condujo también el médico inglés Thomas Sydenham, dedicado a analizar minuciosamente la enfermedad y relacionando las historias clínicas particulares con sus síntomas y con la posibilidad de acotar las señales médicas a enfermedades específicas, según la observación de las constantes. Esto daría lugar a un catálogo bien definido de enfermedades y de indicios reveladores de ellas (REISER, 1990, p. 21). Esta práctica resulta también relevante hasta la actualidad y, para los fines de este artículo, de particular importancia por tener una base idéntica a la que puede constatarse en los 
documentos de conocimiento médico que los colonizadores observaron de la Nueva España y llevaron al viejo mundo.

Respecto a la clasificación de enfermedades en atención a sus síntomas, un ejemplo de ejercicio análogo puede constatarse en el códice de la Cruz Badiano. Nicolás Monardes, el protomédico Francisco Hernández y el padre jesuita José de Acosta dieron a conocer la flora y fauna de la Nueva España. "Todos estos autores (desde su posición) describen y analizan los nuevos hallazgos en materia de medicamentos y los hechos y observaciones de los indígenas configurados en una verdadera medicina aborigen". (Orígenes de la medicina en el Buenos Aires Colonial, 1980, p. 351) Sin duda la política de la duda sugerida por O'Malley había estado presente en esta medicina aborigen.

En el caso de la Nueva Espańa, la mayoría de los médicos se mantenían en la medicina medieval con algunos visos de aprender y adoptar las propuestas de Vesalius. Lo que puede destacarse de la condición de la atención médica a los indígenas es el conocimiento de su medicina; especialmente, de los recursos curativos provenientes de las plantas, aceptados por la corona espańola y llevados a examen por el protomédico Francisco Hernández en el Hospital Real de Naturales, el mismo médico iniciador de la práctica de autopsias entre 1576 y 1581 durante la epidemia del gran cololiztli. (ROMERO, 2003, p. 499-500)

El caso del protomédico Francisco Hernández aporta el juicio médico en igualdad de condiciones entre culturas; lo que prevalece es el interés en la curación con los recursos disponibles pasados por el tamiz de la efectividad para cada caso. Las autopsias, las auscultaciones, la observación de los enfermos, la selección, recolección, aplicación de las hierbas fueron recursos potenciados para la atención médica.

Cabe destacar la pertenencia del médico Francisco Hernández a la institución reguladora de las actividades médicas, a saber: el tribunal del Protomedicato, implantado en España y llevado a la Nueva Espańa para ocuparse de lo relacionado con la salud pública, así como de examinar y conceder las respectivas licencias a aquellos sujetos que de algún modo se iban a consagrar a la medicina o estaban relacionados con ella. Tal es el caso de los cirujanos, barberos, boticarios y el personal paramédico. Casi desde el primer momento de la colonización en Nueva España, el tribunal del Protomedicato inicia labores dictando las "Disposiciones Generales" que fueron reglamentando el quehacer médico, sustentadas generalmente en la legislación española. (SUASTE, 2006, p. 36)

El protomédico Francisco Hernández fue un caso especial, ya que se acercó a la medicina mesoamericana y la reprodujo. A decir de McCleery (2015), el comienzo del control de las licencias médicas puede remontarse a una legislación en Valencia fechada en 1329, cuyo fin fue permitir la práctica 
a los cristianos y excluir a los ajenos. Es aquí donde se evoca la Reconquista ${ }^{2}$ como una de las constantes de la atención médica a pueblos indígenas. Iona Mc Cleery ofrece una indagación acerca de los elementos medievales presentes en la colonia y sostiene, como precursores de los modelos imperiales, a otros modelos medievales. Cita a Bartlett en The Making of Europe:

Los cristianos europeos que navegaron a las costas de las Américas, Asia y África en los siglos XV y XVI provenían de una sociedad que ya era una sociedad colonizadora. Europa, el iniciador de uno de los principales procesos de conquista, colonización y transformación cultural del mundo, también fue el producto de uno. (McCLEERY, 2015)

Los modelos imperiales medievales, antiguos y modernos no tienen en realidad un principio y un fin, ni en la medicina ni en la organización política y social. Lo que seleccionamos para la comprensión son características definitorias, destacadas y predominantes en cada uno de estos modelos que, a su vez, cohabitan en los tiempos con denominaciones variables y significados también mudables. Veamos el caso del origen de las enfermedades.

Desde la Antigüedad, la idea de los síntomas expresados en enfermedades ocasionadas por entidades malignas había sido propuesta por Platón y actualizada por el médico suizo Paracelso, quien recorrió toda Europa practicando la medicina. Sostuvo Paracelso que las enfermedades no sólo eran manifestaciones de desequilibrios humorales, sino que eran entidades reales que diferían en su composición concreta y brotaban de causas específicas. (REISER, 1990, p. 19)

De la medicina medieval, es preciso señalar que estaba fundamentada en la idea del equilibrio entre humores. Se denominaba así a las sustancias en el cuerpo identificadas como bilis negra, bilis amarilla, sangre y flema, y se atribuía a cada una un temperamento. Así, la predominancia de sangre se refleja en personas sociables, la de flema corresponde a carácter calmado, bilis negra a melancolía y bilis amarilla a un carácter colérico. A su vez, cada una de ellas se vinculaba a un elemento natural: bilis negra a la tierra, bilis amarilla al fuego, flema al agua y sangre al aire. "Su armoniosa mezcla aseguraba la salud; una ruptura de su equilibrio (...) produciría la enfermedad (...) una perturbación humoral o destemple. Por regla general (...), la terapia de enfermedades tan graves no iba dirigida a las enfermedades como entidades,

2 La Reconquista cristiana supuso el criterio de pureza de sangre como requisito para pertenecer a la élite y fue el pretexto argüido para expropiar propiedades no-cristianas. De esta manera, el no pertenecer a la sangre cristiana garantizaba la exclusión y el "derecho" -de los cántabros en la Reconquista cristiana y de los cristianos en la Conquista española- a someter, denigrar, ignorar, subestimar, robar, despojar (STANLEY \& STEIN, 1987, p. 58) y negar el derecho de los "otros" (de los no-ellos) a tener sus propias formas de curación, en este caso. 
sino que, antes bien, tendía a restaurar la armonía en las funciones corporales del paciente: los médicos trataban fiebres, flujos e hidropesías, antes que enfermedades particulares" (REISER, 1990, p. 19). En cuanto al espacio donde se proporciona la atención:

El centro de la práctica médica en los siglos XVII y XVIII era el hogar, fuese del paciente o del médico. La atención institucional en lugares como hospitales era, principalmente, para quienes no tenían medios financieros ni familia, amigos o domésticos que les cuidaran; o para los enfermos que habiendo probado todo lo demás, esperaban encontrar ayuda fuera de su casa. (REISER, 1990, p. 13)

(...) En ocasiones, si un paciente necesitaba prolongada atención médica y vivía lejos del médico, acaso se quedara alojado días y aún semanas en casa de éste. Mas para ahorrar a los pacientes y a ellos mismos la dificultad y las molestias del viaje, los médicos a menudo diagnosticaban enfermedades y prescribían tratamientos por correo. (REISER, 1990, p. 17-18)

De la misma manera que en Europa, en la Nueva España una de las funciones de los hospitales era albergar a personas en situación menesterosa. El establecimiento de "hospitales de indios" tuvo por "objetivo atender a personas de escasos recursos y forasteros” (CHÁVEZ, 2008, p. 35).

Insinuamos ahora la primera concurrencia: para los mayas, los lugares o espacios inciden en el proceso de curación. Generalmente, los recursos naturales (raíces, cortezas, plantas) recolectados o sembrados y empleados para sanar, eran llevados a casa del enfermo o del médico maya. En casos graves, los recursos se llevaban a cuevas o caminos (TV UNAM, 2007). En este sentido encontramos similitud en el sitio de la práctica médica. Si bien en la medicina europea no hay implicada una sacralización (que sí la hay en la medicina maya), ambas se llevaron a cabo en el hogar del médico o del paciente, y si se escudriñaran las coincidencias, si se mirara el ser del otro con el valor del propio, se podría encontrar, aceptar y valorar la coincidencia.

La segunda coexistencia es la de la concepción de la enfermedad producida por un desequilibrio en entidades internas del cuerpo. En el caso de la visión europea, la idea de los humores corporales contenidos en la bilis negra, bilis amarilla, flema y sangre son los que determinan el carácter y la condición de salud del cuerpo. Por lo que hace a la medicina maya, el equilibrio en las materias contenidas en el cuerpo.

El doctor Velázquez (2019) explica que, para los mesoamericanos, el cuerpo humano no es algo fijo; es mutable y se constituye de partes numinosas o de materia ligera, es decir, entidades anímicas que se esparcen por todo el cuerpo (aunque se concentren en un centro anímico). Estas entidades tienen 
personalidad y voluntad propias y se mueven a voluntad del ser humano que las posee; se las denomina kihn, o'hlis y wahyis.

Existen otras dos fuerzas que son impersonales, no se mueven a voluntad del ser humano que las posee y habitan tanto en el interior como en el exterior del cuerpo humano, y se vinculan con fuentes externas que les permiten regenerarse y renovarse. Es a lo que se le ha llamado el hálito de la respiración y, como puede constatarse biológicamente, se nutre de lo externo y lo interno. Cuando este hálito se termina, se dice que se perdió su blanco, puro aliento vital (sak ik'aal) y se hizo nada su puro y blanco aliento vital. La muerte es la separación de todos los componentes del cuerpo, todas las materialidades y los componentes etéreos (VELÁZQUEZ, 2019).

Es notorio un sustrato común en las interpretaciones del cuerpo entre médicos del siglo XVII y el cuerpo para los mayas; lo común entre ambas es el asignar entidades inherentes a todos los cuerpos. En la medicina occidental del siglo XVII, estas entidades eran llamadas humores y solo poseían una condición de materialidad. En cambio, las entidades del cuerpo maya no eran solo materiales e inexplicables, sino que se vinculaban con lo externo y, además, tenían una clasificación o tipología: unas eran volitivas, otras no. Esta idea sería, desde una posición no-colonialista, de una complejidad y una riqueza valiosísimas, y por su complejidad, de mayor valor e interés que la sola explicación de los cuatro humores, centrada en algunas observaciones anatómicas características de esa época. Pero la colonialidad no permitió su conocimiento y su reconocimiento, como todavía en la actualidad no existe dentro del ámbito médico, aunque sirva para explicar y comprender los procesos de salud-enfermedad-atención, y para curar al igual que la medicina institucionalizada.

Este pensamiento análogo entre los humores y las entidades de los cuerpos pudo haber sido conocido o reconocido por los pueblos mesoamericanos y asumido para la comprensión de los padecimientos, pero no lo fue abiertamente por los médicos europeos. Podemos afirmar que, contrapuesto a lo que se creía respecto a los fundamentos solo materiales de la enfermedad, recientemente se conocen casos de médicos y experiencias de atención con medicinas alternativas a partir de una etiología de la enfermedad no solo material, sino por una condición llamada también inmaterial, espiritual o emocional denominada psicosomática ${ }^{3}$, en la cual la enfermedad no se atribuye a un agente material externo sino a los pensamientos.

Nos referimos, por ejemplo, a la neuróloga Suzzane O'Sullivan, cuya carrera facultativa dentro del National Hospital for Neurology and Neurosurgery en Gran Bretańa le permite afirmar que uno de cada tres pacientes atendidos

3 Se califica como psicosomático al trastorno psicológico que genera un efecto físico, provocando alguna consecuencia en el organismo. 
en una clínica neurológica tiene enfermedades psicosomáticas. En entrevista declaró que uno de los problemas de la formación médica especializada es el diagnóstico de enfermedades por partes (rodilla, cerebro, pensamientos) sin ver más allá de ello. Sostiene que las especialidades son necesarias pero "han de mirar a la persona como un todo" (O'SULLIVAN, 2016).

Actualmente en Cuba se plantea la atención combinada entre medicina convencional (o medicina europea) y medicina tradicional china:

Las técnicas de la Medicina Tradicional Natural MTN pueden ser aplicadas en situaciones de emergencia y desastres, ya que ofrecen una amplia gama de posibilidades terapéuticas menos dañinas y más eficientes desde el punto de vista económico, por el ahorro de medicamentos químico-industrial. Además propicia la solución de los problemas de salud de las poblaciones con sus tradiciones y sistemas ecológicos; así como prepara al individuo para autosolucionar algunas de sus dolencias, tanto en la esfera psíquica como física, y a su vez permite la práctica de estilos de vida más integradores, en el camino hacia la salud. (ÁLVAREZ, MONTES DE OCA, FERREIRO, 2014, p. 517)

A este respecto, es de resaltar que "la enfermedad para los indígenas implicaba desórdenes que afectaban tanto al cuerpo como al alma y, sobre todo, las relaciones que éstos sostienen con el universo". (VICTORIA, 2004, p. 146)

La colonialidad impide la exploración de estas acepciones, no por su fundamento, posibilidad o imposibilidad, sino por su origen y por el origen de quien las piensa, dentro de un orden dominante en el que el poder, el saber y el ser sólo son propios de una cultura que se ostenta como dominante.

Atención hospitalaria a pueblos indígenas en la Colonia

Respecto a los centros hospitalarios en México, independientes de la conquista y la colonia, Clavijero mencionó la existencia de una casa para enfermedades incurables en el palacio de Moctezuma II, un hospicio ubicado al lado del Templo Mayor. (SUASTE, 2006, p. 30)

Antes de la llegada de los españoles, los aztecas ya habían vivido y combatido epidemias con su propia medicina. Hambrunas, catarros, problemas intestinales, enfermedades de la piel, entre otros padecimientos generalizados con las épocas de sequías, fueron tratados con la medicina que los aztecas aprendieron y retomaron de los pueblos conquistados. (VICTORIA, 2004, p. 148)

Es indudable el maltrato, trabajos forzados, condiciones de explotación, desnutrición, además de enfermedades contagiadas a los pueblos indígenas durante la colonia, así como la merma poblacional que con ellos se produjo. 
Una de las respuestas a tal situación fue la edificación de hospitales, el primero por el propio Hernán Cortés con el trabajo y respaldo de órdenes religiosas (VICTORIA, 2004, p. 149). La interpretación de Josefina Muriel en cuanto a los móviles y la relevancia de las fundaciones de hospitales durante el siglo XVI y XVII en la Nueva España es el surgimiento de una "mística religiosa que trajo consigo la obra misional cuya fuerza transformó a orgullosos conquistadores, abusivos encomenderos y ambiciosos comerciantes en humildes y generosos hospitaleros que dieron sus vidas al servicio de los pobres enfermos" (SUASTE, 2006).

A decir verdad, sería difícil determinar si lo que motivó al "movimiento hospitalario" fue una mística religiosa o una necesidad de mantener la mano de obra que sostenía al sistema colonial, ya que, como señala el Chilam Balam de Chumayel:

No había entonces enfermedad; no había dolor de huesos; no había fiebre para ellos; no había viruelas; no había ardor de pecho; no había dolor de vientre; no había consunción (...) No fue así lo que hicieron los extranjeros cuando llegaron aquí. (STANLEY \& STEIN, 1987, p. 34)

Por el contrario, algunas fuentes apuntan a la economía minera y el establecimiento del sistema de hacienda como una de las condiciones que diezmó a la población indígena por las características del trabajo ${ }^{4}$. Fueron tratados como animales de carga y sometidos a un trabajo excesivo, además de la ruptura de la sociedad comunal a la individual. En México, el descenso calculado entre 1525 y 1605 fue de 24 millones de personas. (STANLEY \& STEIN, 1987, p. 34-40)

Ley I de Indias que decía: “(...) en todos los pueblos de españoles e indios se funden hospitales, donde sean curados los pobres enfermos y se ejercite la caridad cristiana”. Esa institución debía situarse cerca de una iglesia, ser cuidada por el Cabildo y tener como patrono al de la ciudad. (PÉRGOLA, 2014, p. 45)

\footnotetext{
4 En 1970, Stanley \& Stein postularon las enfermedades epidémicas, el trabajo excesivo y la consecuente debilitación física como causas de "uno de los descensos demográficos más desastrosos en la historia universal" ocurrido en entre el siglo XVI y XVII en las colonias españolas (1987, p. 40). Más recientemente, Robert McCaa reitera que existe un consenso sobre las causas de la catastrófica declinación poblacional y "sobre el hecho de que las epidemias, la explotación y las disrupciones ecológicas fueron los principales agentes". El autor se remite a las Relaciones Geográficas de los nativos, escritas a finales del siglo XVI, donde el exceso de trabajo se menciona en muchos casos (al igual que la intervención divina) y concluye: Existe un acuerdo sobre el hecho de que una catástrofe demográfica ocurrió y que las enfermedades epidémicas fueron un factor determinante para que la mortalidad se desatara, empezando, en México central, con la viruela de 1520. Pero el rol de las enfermedades no puede ser entendido sin tener en cuenta el cruel tratamiento a que se sometió a la masa de la población nativa (migración forzada, esclavitud, demandas laborales abusivas y tributos exorbitantes). (MCCAA ROBERT, 1995, p. 127-132)
} 
En este contexto (a consecuencia de una obra misional como refiere Josefina Muriel, o impulsados por sostener la economía colonial basada en el trabajo de los indígenas), por iniciativa de Hernán Cortés se fundó un hospital para la atención de los indios afectados por las epidemias, con capacidad para hasta cuatrocientos pacientes. Fue fray Pedro de Gante quien realizó las gestiones ante el emperador Carlos $\mathrm{V}$ y obtuvo la Cédula Real para su construcción (ROMERO, 2003, p. 496).

Así como los frailes gestionaron la construcción del hospital, se mantuvieron en la atención, con la ventaja de conocer el idioma de quienes acudían. Hablaban náhuatl y otomí, escuchaban al paciente y luego lo remitían con el médico. Se sabe también que, en los hospitales fundados, se brindaba una atención mixta de médicos españoles y médicos indígenas apoyados por los frailes.

La atención médica híbrida es a mi juicio un elemento favorable al cuidado de la salud de los pacientes indígenas. Si bien la enfermedad había sido generada y agudizada por condiciones ajenas a los pueblos e inauguradas por los colonizadores, el reconocimiento implícito o explícito de las concepciones médicas y tratamientos fue incorporado a las instituciones de salud europeas y significó en algún rubro un alivio a los pacientes, desde la perspectiva de ser sanados con sus propios recursos e integrando su cosmovisión de la salud y la enfermedad. Esta "concesión" es útil y es necesario reincorporar a la atención médica hospitalaria de los pueblos mesoamericanos, que siguen acudiendo a hospitales y enfrentándose a visiones de la atención muy distintas a las suyas.

Los doctores Andrés Romero Huesca y Julio Ramírez Bollas, en una investigación acerca de la atención médica en el Hospital Real de Naturales, señalan que en este lugar podían realizarse procedimientos no permitidos en la península -autopsias- y que la idea que sostenía este permiso era la de los indígenas como seres inferiores a los españoles. Para el procedimiento sólo se requería la autorización del virrey. Con estos supuestos, se fundó la Real Escuela de Cirugía en México, que inició sus funciones el 3 de febrero de 1770 y se constituyó como un ejemplo de los avances en la interpretación médica que pasó del estudio teórico al clínico y práctico. (ROMERO, 2003, p. 501)

El problema de la colonialidad es en sí su incapacidad para equipararse al otro, su creencia de superioridad y el sostener esa creída posición como la base de su existencia y su sistema, con las terribles e insuperables consecuencias de racismo, clasismo, desigualdad, violencia simbólica y física que ello entrańa. Sin embargo, por encima, por abajo y transversal a esas creencias dañinas a la pluralidad social, la vida, las prácticas y, en este caso, la ciencia médica, toman curso propio e interpretaciones y alcances no-coloniales, que suman al conocimiento que luego deberá abrirse a descolonizarse y expandirse a todos los grupos antes excluidos. 
Ejemplo de los alcances en la colonialidad, al margen de ella, fue la creación de hospitales de naturales y la Real Escuela de Cirugía, creada en 1768 en el seno del Hospital Real de Indios de México, que se apegó a lo que en su momento establecieron las autoridades para la apertura del Colegio de Cirugía de Cádiz y el de Barcelona, en España. (SUASTE, 2006, p. 36)

Al igual que en España y la Nueva España, en Inglaterra muchos médicos en el siglo XVIII continuaron los esfuerzos de Sydenham por clasificar las enfermedades.

(...) William Cullen, profesor de medicina de la escuela de Edimburgo (...) declaró que el objetivo de la clasificación de las enfermedades debía ser definir los "patognomónicos", síntomas que eran "tan propios de cada enfermedad que tan solo por ellos podemos rápida y seguramente distinguirla de todas las demás. Sydenham, Sauveges y Cullen basaron sus clasificaciones en síntomas mostrados por pacientes vivos. (REISER, 1990, p. 22)

La Real Escuela de Cirugía se distinguió por impartir cátedras de anatomía, fisiología, operaciones, clínica quirúrgica y medicina legal, en contraste con la formación teórica preferida en la Real y Pontificia Universidad de México. Esta base empírica y directa de la ciencia médica ya había estado presente en la medicina mesoamericana, pero, como se señaló anteriormente, no es propio de la colonialidad el aceptar la capacidad del otro para desarrollar algo equivalente a lo suyo. A decir de Santiago Castro-Gómez (2005): "La Ilustración no solo planteaba la superioridad de unos hombres sobre otros, sino también la superioridad de unas formas de conocimiento sobre otras". (RUIZ, 2009, p. 144)

La conveniencia y arbitrariedad de la supuesta superioridad desde la época colonial (el Medioevo en caso de conceder a McCleery un origen de la colonialidad en la medicina) siguen reproduciéndose en diferentes ámbitos. El núcleo es que nada de lo que sea o haga la cultura sometida tendrá un valor equiparable a la cultura colonizadora; así se observó en la medicina y así puede constatarse tanto en la medicina como en otros ámbitos. Para muestra, quiero terminar con un fragmento de la película Raíces, basada en el cuento "Nuestra Señora de Nequetejé" de Francisco Rojas González.

En la historia interviene una investigadora francesa que quiere estudiar a los indígenas chamulas en Chiapas. Al estar en convivencia con el pueblo, se entera de que los niños recién nacidos tienen dos nombres: el nombre de pila con el que se bautizan en la iglesia católica, y el nombre del nagual, que es el animal que los cuidará durante su vida. Dicho nombre lo determinará el animal que deje su huella en las cenizas, que se colocan afuera de la casa para la espera del animal que imprimirá ahí su huella, ya sea antes o después del nacimiento del niño. La investigadora, con nula precaución, llega a la casa 
de los padres del recién nacido en su bicicleta y pasa la rueda por encima de las cenizas, por lo que el nombre del niño es Anselmo Bicicleta. Más tarde, la misma investigadora lleva a la comunidad algunas obras de arte europeo para "medir" o conocer la capacidad de apreciación del arte de este pueblo. En este contexto y después de terminar su "trabajo de campo" y regresar a la comunidad, donde la recibe el sacerdote, dice lo siguiente:

Investigadora: Los indios no están capacitados para entender las grandes obras de nuestra gran cultura.

Sacerdote: Muchas veces, llamamos salvaje a lo que no entendemos.

Investigadora: Les dan nombres absurdos a sus hijos.

Sacerdote: Sí, aquí las gentes se llaman Mirlo (un ave) o Bicicleta, pero en otras partes del mundo civilizado se llaman Pedro, que quiere decir piedra o López que quiere decir Lobo. (ALAZRAQUI, 1953)

Así son los coloniales, incapaces de mirarse en el otro, queriendo medir la luz con una regla, con una báscula o un reloj para invalidar lo ajeno, usando instrumentos no pertinentes.

En cuanto a la actualidad de pacientes indígenas hospitalizados, recupero el texto escrito por un poeta tojolabal:

EN EL HOSPITAL. Escuchen mis hermanos // el cuento que les digo // las cosas que yo veo // estoy en Comitán. Enfermo estoy aquí // en este hospital // así lo veo bien // el modo de su hacer. Pues en el hospital // enfermos muchos hay // de diferentes pueblos // con males muy diversos. Hermanos nuestros sufren // muy fuerte es su dolor // y otros sin dolor // según la enfermedad. En este hospital // si llega un patrón // con gusto lo reciben // pues sí se caen bien. Si llega un hermano // que es tojolabal; // andamos bien perdidos // dentro del hospital. La lengua no entendemos // que los doctores hablan // tampoco nos entienden // ni nuestra enfermedad Y si no nos entienden // tampoco nos respetan // su corazón les dice // que no sabemos nada. La lengua de nosotros // no quieren aprender // porque ellos no respetan // así como hablamos. Escuchen mi palabra // del corazón nació // los meros ignorantes // son ellos de verdad. En este mundo, digo // iguales son los cuerpos // hermanos somos todos // de una humanidad. Hay blancos y morenos // bambaras, chinos, indios // hermanos somos todos // de una humanidad. Por ello ya nosotros // debemos aprender // la lengua que es de ellos // que nos respeten ya. También les toca a ellos // el mismo aprender // la lengua que es la nuestra // hermanos, pues, seremos. Hermanos, me despido // y que les vaya bien // y no les pase nada // por sendas y veredas. Nos vemos, nos hablamos, // en otra ocasión // platicaremos cómo // estamos por acá. El poeta murió pocos meses después de leucemia aguda. (LENKERSDORF, 1999) 
ALAZRAQUI, B. Raices [Drama indigenista]. Teleproducciones S.A., 1953.

ÁLVAREZ, F. E, MONTES DE OCA, M., FERREIRO, Y. Diagnóstico y tratamiento de la medicina tradicional y natural, como alternativa en emergencia y desastres. Revista Cubana de Medicina Militar, v. 43, n.4, 2014, p. 515-552.

CHÁVEZ, G. M. Trayectoria de los centros de salud europeos y primeras luces de la medicina moderna en el Yucatán colonial. Peninsula, n. 3, 2008, p. 35-63.

GARCÍA, V. M. Medicina y sociedad. México: Fondo de Cultura Económica, 1994.

LENKERSDORF, Carlos. (Org.) Indios somos con orgullo. poesia maya-tojolobal (Literatura indigena bilingue). Ciudad de México: UNAM, 1999.

MCCAA, R. ¿Fue el siglo XVI una catástrofe demográfica para México? Una respuesta basada en la demografía histórica no cuantitativa. Cuadernos de Historia, n. 15, 1995, p. 123-136.

McCLEERY, I. What is "colonial" about medieval colonial medicine? Iberian health in global context. Journal of Medieval Iberian Studies, v. 7, n. 2, 2015, p. 151-175. doi 10.1080/17546559.2015.1077390

O'SULLIVAN, S. Uno de cada tres pacientes tiene una enfermedad imaginaria [Elmundo.es]. Recuperado de https://www.elmundo.es/ salud/2016/01/29/56aa663eca4741193b8b4692.html, 29 enero 2016.

PÉRGOLA, F. Los hospitales coloniales. Revista Argentina de Salud Pública, v. 5, n. 21, 2014, p. 45-46.

REISER, S. J. La medicina y el imperio de la tecnología. México: Secretaría de Salud, Fondo de Cultura Económica, 1990.

ROMERO, H. A; RAMÍREZ B, J. La atención médica en el Hospital Real de Naturales. Cirugia y Cirujanos, v. 71, n. 6, 2003, p. 496-503.

RUIZ, S.M. La Ilustración Hispanoamericana. In: DUSSEL E. (ed.), El pensamiento filosófico latinoamericano, del Caribe y latino (1300-2000): Historia, corrientes, temas y filósofos, México: Siglo XXI-Centro de Cooperación Regional para la Educación de los Adultos en América Latina y el Caribe, 2009, p. 143-153.

STANLEY, J., \& STEIN, B. H. La herencia colonial de América Latina. 19a ed. México: Siglo Veintiuno, 1987.

SUASTE GÓMEZ, E. Antecesores en el devenir de la técnica y medicina en México. CINVESTAV, v. 25, n. 3, 2006, p. 25-37. 
TV UNAM. La historia de la medicina en México. Cap.: II, La medicina maya. (AstraZeneca). México: UNAM, 2007.

VERDESIO, G. Colonialidad, colonialismo y estudios coloniales: Hacia un enfoque comparativo de inflexión subalternista. Tabula Rasa, v. 29, 2018, p. 85-106. doi: $10.25058 / 20112742 . n 29.05$

VELÁZQUEZ, G. E. Conferencia magistral. 5 agosto 2019.

VICTORIA, J. A. M. 500 años de salud indígena. México, D.F: Cámara de Diputados, 2004.

Mariana Ramírez Manzano. Alumna de la Maestría en Ciencias Sociales de la Universidad Autónoma de la Ciudad de México. Estudia las relaciones entre pueblos indígenas e instituciones públicas y su dimensión política, económica y cultural, en perspectiva histórica y contemporánea.

E-mail: mramirezmanzano@gmail.com 